\title{
LARGE CONGENITAL LEFT ATRIAL APPENDAGE ANEURYSM IN AN ADULT
}

\author{
Neeraj Shah ${ }^{1}$, Sandra Abramson ${ }^{2}$, Katie Hawthorne ${ }^{2}$, William Gray ${ }^{2}$, Paul Coady ${ }^{2}$, Eric \\ Gnall $^{2}$, Scott Goldman ${ }^{2}$, and Roberto Rodriguez ${ }^{3}$ \\ ${ }^{1}$ East Carolina University \\ ${ }^{2}$ Lankenau Medical Center \\ ${ }^{3}$ Lankenau Hospital
}

May 6, 2020

\begin{abstract}
Left atrial appendage aneurysm is a rare cardiac abnormality predisposing to atrial arrhythmias and thromboembolic phenomena. When discovered, surgical management is the treatment of choice. Here we describe a case of incidentally discovered large congenital left atrial appendage aneurysm in a young adult that was treated with surgical resection.
\end{abstract}

\section{History}

A 19-year-old female with no significant medical history presented with palpitations. A day before presentation she reported blunt chest trauma when someone fell onto her chest. Following this, she continued to have mild chest discomfort and intermittent palpitations. She noticed that her Apple Watch showed heart rate over $200 \mathrm{bpm}$ and this prompted her to come to the emergency room (ER). In the ER, she was found to have supraventricular tachycardia (SVT) with heart rate $218 \mathrm{bpm}$ unresponsive to vagal maneuvers (Figure 1A). Ultimately, sinus rhythm was restored after intravenous administration of adenosine and beta blockers (Figure 1B). She reported a short-lasting episode of palpitations three months prior to this presentation that resolved on its own.

\section{Investigations}

Chest X-ray showed cardiomegaly with abnormal contour of left heart (Figure 2). Given history of chest trauma, a transthoracic echocardiogram (TTE) was performed which revealed a large echolucent structure lateral to the left heart (Figure 3A-C, Video). Contrast echocardiography showed that structure was contiguous with the left atrium (LA) [Figure 3C].

\section{Diagnosis}

At this point, differential diagnosis included intracardiac tumor, pericardial cyst or mediastinal mass. Contrast echocardiography revealed free communication between the lateral echolucent structure and LA (Figure 3C) suggesting that this was an intra-cardiac structure. Computerized tomography (CT) chest with intravenous contrast was then ordered for definitive diagnosis. CT chest revealed a large LAAA measuring 6.2 $\mathrm{cm}$ transverse $\mathrm{x} 6.4 \mathrm{~cm}$ anteroposterior x $11.0 \mathrm{~cm}$ cephalocaudal (Figure 4). The neck of LAA measured 3.3 $\mathrm{cm}$. CT scan showed thickening of the LAA wall and some opacities within the LAAA cavity suggesting presence of thrombus. Aside from the LAAA, there were no pertinent cardiac abnormalities.

\section{Management}


Given the large LAAA with concomitant arrhythmias and risk of thromboembolism, the patient was advised to undergo surgery for removal of the aneurysm. There is potential risk of a perioperative thromboembolic event with minimally invasive surgical approach due to less controlled manipulation of the aneurysm during resection. The size of aneurysm and presence of thrombus by CT determined our surgical approach to be median sternotomy with cardiac arrest during cardiopulmonary bypass (CPB). This approach would give us the most controlled and low risk scenario during resection. The patient was placed on CPB after direct cannulation of ascending aorta and cannulation of right atrium via right common femoral vein approach. The aorta was cross clamped and Del Nido antegrade cardioplegia was delivered to the aortic root. Once the heart was decompressed, the large LAAA was visualized (Figure 5) and careful dissection was performed along the neck of the aneurysm. A $50 \mathrm{~mm}$ AtriCure clip was placed across base of the LAA and the aneurysm was resected (Figure 5, Video 1). Histopathological examination confirmed a thin walled LAAA.

\section{Follow up}

Postoperative course was uneventful, and the patient was discharged home four days later on low dose aspirin and beta blocker therapy. At the time of one-month follow up, the patient was doing well without any issues.

\section{Discussion}

LAA aneurysm is an extremely rare entity. It has slight female preponderance and is usually diagnosed in the third decade of life. ${ }^{1}$ It is often discovered incidentally on imaging studies in the absence of symptoms. ${ }^{2}$ LAAA is classified as congenital or acquired with $>90 \%$ of the cases being congenital thought to arise from congenital dysplasia of the pectinate muscles. ${ }^{2}$ It is rarely associated with other cardiac anomalies such as atrial or ventricular septal defect. ${ }^{1,2}$ Acquired causes of LAAA include mitral valve disease or elevated left atrial pressure. ${ }^{2}$

Most common symptoms associated with LAAA include palpitations and dyspnea. ${ }^{1}$ Other symptoms include chest pain and systemic thromboembolism. Significantly enlarged LAA provides a substrate for atrial or supraventricular tachyarrhythmias such as SVT, atrial tachycardia, fibrillation or flutter, which manifest as palpitations. Aneurysmal dilatation of the LAA results in stasis of blood and predisposes to thrombus formation. Additionally, these patients are at high risk of developing atrial fibrillation or flutter which further increases thromboembolic risk. ${ }^{1}$ The incidence of stroke or systemic thromboembolism in LAAA ranges from $6-16 \% .^{1,2}$ Rarely, a large LAAA compresses the coronary arteries resulting in myocardial ischemia presenting as angina. ${ }^{3}$

LAAA is usually diagnosed with multimodality imaging including echocardiography, CT and MRI. Notably, in large LAA aneurysms, chest X-ray is abnormal with cardiomegaly and enlarged left heart border [Figure $2] .{ }^{1}$ TTE is useful as an initial tool for evaluation of LAAA. ${ }^{4}$ Transesophageal echocardiography is valuable for thrombus detection. ${ }^{5}$ Contrast echocardiography aids in confirming the diagnosis as well as ruling out thrombus formation. ${ }^{4} \mathrm{CT}$ diagnoses LAAA with a high degree of accuracy and offers additional advantage of assessing coronary anatomy. ${ }^{2}$

Surgical resection remains the cornerstone of therapy for LAAA. Early surgical intervention is recommended given the risk of thromboembolic complications. ${ }^{6}$ Median sternotomy is the preferred surgical approach in majority $(85 \%)$ of the cases. ${ }^{2}$ Other less invasive approaches such as left lateral thoracotomy, mini-thoracotomy and endoscopic resection have been described ${ }^{7-9}$, however, these approaches are more suited for smaller aneurysms without intra-aneurysmal thrombi. Additionally, there is risk of incomplete closure with minimally invasive approach. ${ }^{9}$ Therefore, for large LAAA (such as in our case) median sternotomy with CPB is recommended to minimize the risk of thromboembolic complications resulting from dislodgement of small thrombi during surgical manipulation ${ }^{1}$ and to provide a clear field to accurately and completely resect the aneurysm and avoid coronary artery injury [e.g. left circumflex artery located at the base of LAA] ${ }^{6}$ After successful surgical treatment, the prognosis is excellent with freedom from recurrent symptoms, arrhythmia and thromboembolic events reported in long term follow-up. ${ }^{1,2}$

\section{Conclusions}


LAAA is a rare but clinically important entity associated with high risk of thromboembolic complications. Surgical resection is the treatment of choice.

\section{References}

1. Aryal MR, Hakim FA, Ghimire S, et al. Left atrial appendage aneurysm: a systematic review of 82 cases. Echocardiography.2014;31(10):1312-1318.

2. Wang B, Li H, Zhang L, et al. Congenital left atrial appendage aneurysm: A rare case report and literature review. Medicine (Baltimore). 2018;97(2):e9344.

3. Frambach PJ, Geskes GG, Cheriex EC, Wellens HJ, Penn OC. Giant intrapericardial aneurysm of the left atrial appendage. Eur Heart J. 1990;11(9):848-853.

4. Harland DR, Suma V, Muthukumar L, Port SC, Werner PH, Tajik AJ. Giant Congenital Left Atrial Appendage Aneurysm Presenting With Recurrent Supraventricular Tachycardia and Chest Pain. CASE (Phila).2019;3(3):129-132.

5. Kwan CM, Tsai LM, Lin LJ, Yang YJ, Chen JH. Congenital left atrial appendage aneurysm with thrombus formation: diagnosis by transesophageal echocardiography. J Clin Ultrasound. 1993;21(7):480-483.

6. Jiang B, Wang X, Liu F, Song L. Left atrial appendage aneurysm.Interact Cardiovasc Thorac Surg. 2019.

7. Victor S, Nayak VM. Aneurysm of the left atrial appendage. Tex Heart Inst J. 2001;28(2):111-118.

8. Kiaii B, Doll N, Kuehl M, Mohr FW. Minimal invasive endoscopic resection of a giant left atrial appendage aneurysm. Ann Thorac Surg. 2004;77(4):1437-1438.

9. Kim YW, Kim HJ, Ju MH, Lee JW. The Treatment of Left Atrial Appendage Aneurysm by a Minimally Invasive Approach. Korean J Thorac Cardiovasc Surg. 2018;51(2):146-148.

\section{Figure Legends}

Figure 1: A) Initial electrocardiogram showing supraventricular tachycardia at $218 \mathrm{bpm}, \mathrm{B})$ Repeat electrocardiogram after administration of IV adenosine and metoprolol showing normal sinus rhythm with sinus arrhythmia.

Figure 2: Anteroposterior chest X-ray showing abnormal contour of the left heart.

Figure 3: Transthoracic echocardiography (TTE) findings. A) Short axis TTE view at level of mitral valve showing a large lateral echolucent structure, B) Apical 4 chamber TTE view showing large echolucent structure adjacent to left atrium (LA), C) Administration of Definity echo contrast confirming this to be an intra-cardiac structure communicating with the LA.

$\mathrm{LA}=$ left atrium, $\mathrm{LV}=$ left ventricle, $\mathrm{RA}=$ right atrium, $\mathrm{RV}=$ right ventricle, $\mathrm{LAA}=$ left atrial appendage.

Figure 4: Coronal (panels A and C) and axial (panel B) computerized tomography (CT) views showing the left atrial appendage aneurysm. $\mathrm{LA}=$ left atrium, $\mathrm{LV}=$ left ventricle, $\mathrm{RA}=$ right atrium, $\mathrm{RV}=$ right ventricle, $\mathrm{LAAA}=$ left atrial appendage aneurysm, Ao $=$ Aortic valve, RUPV $=$ right upper pulmonary vein, $R L P V=$ right lower pulmonary vein, $L U P V=$ left upper pulmonary vein.

Figure 5: Intraoperative images during left atrial appendage aneurysm (LAAA) resection. A) Initial appearance of the large LAAA (white arrow) seen on lateral aspect of the heart, B) LAAA (labeled) after full cardiopulmonary bypass (CPB) and decompression of the heart, C) Placement of a $50 \mathrm{~mm}$ AtriCure Clip (marked by asterisk*) at the base of the LAAA, D) Resection of the LAAA after placement of AtriCure Clip.

Video 1: Intraoperative video of left atrial appendage aneurysm (LAAA) resection. 


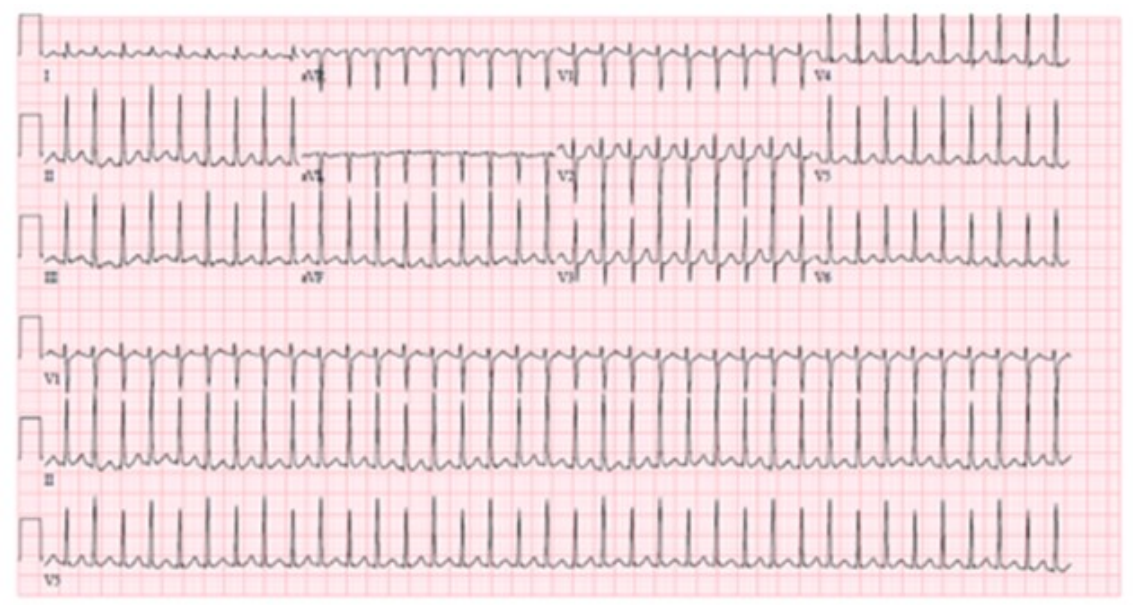

A

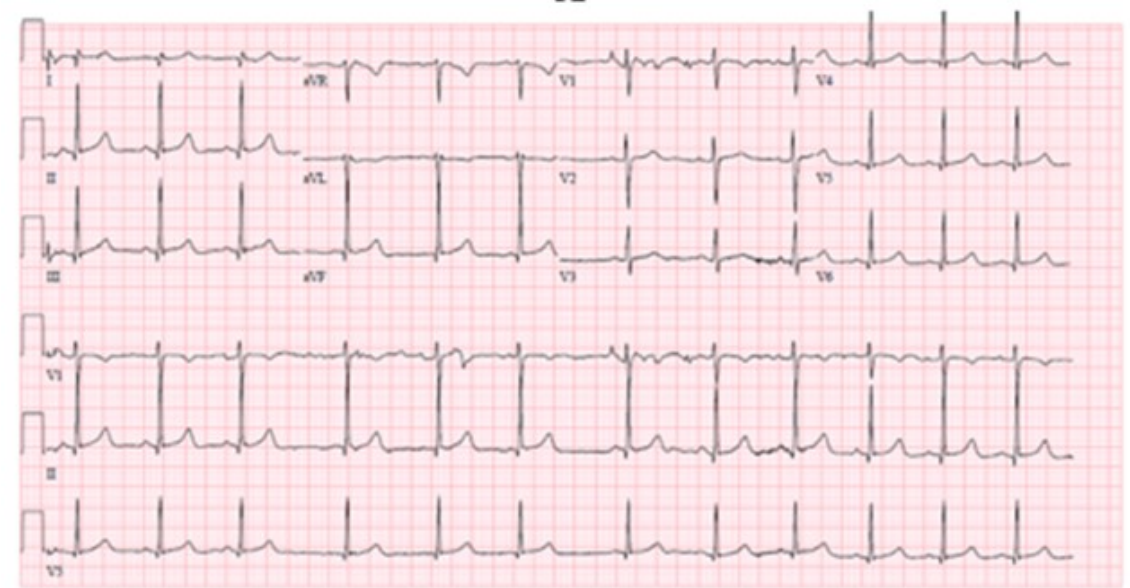

B 

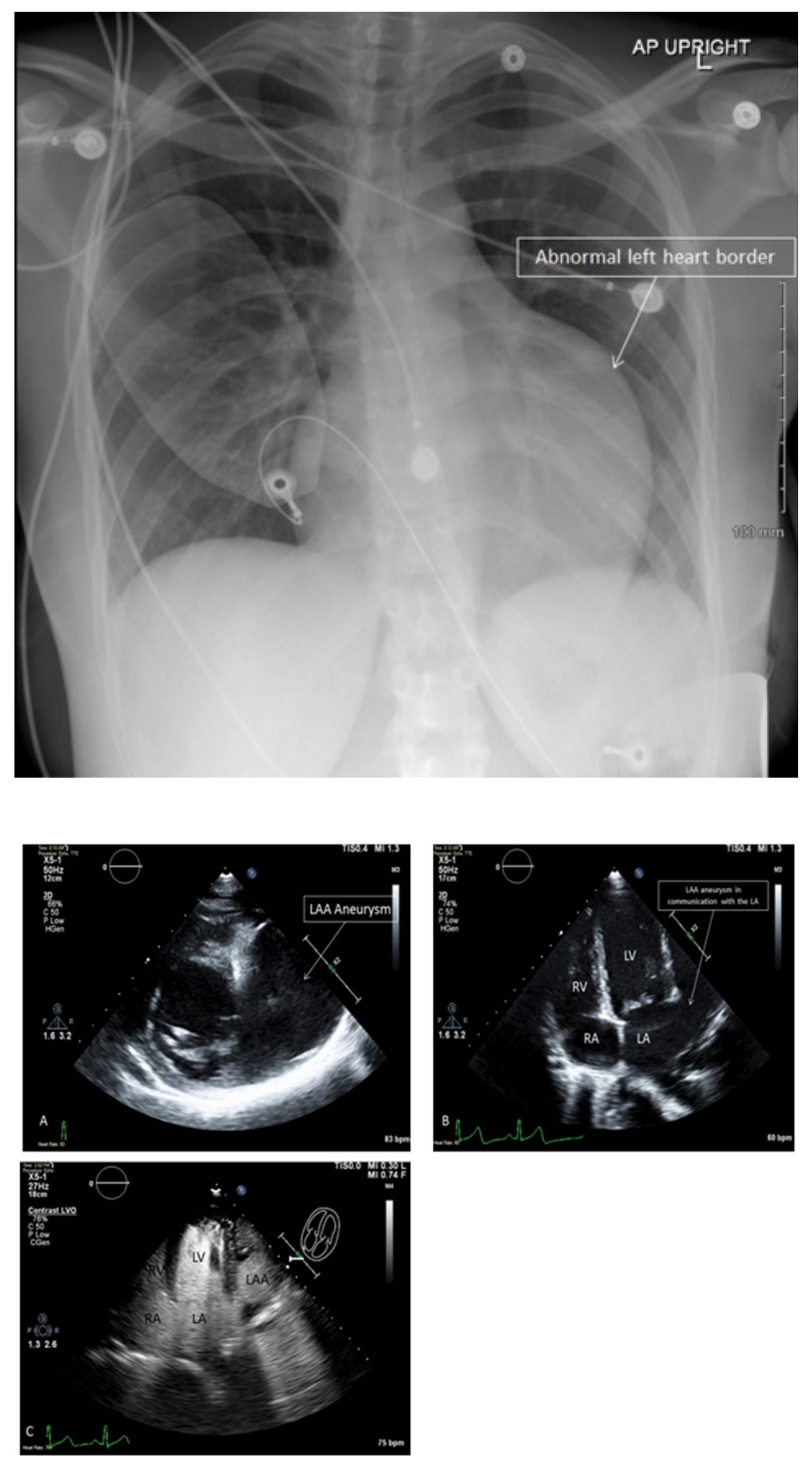

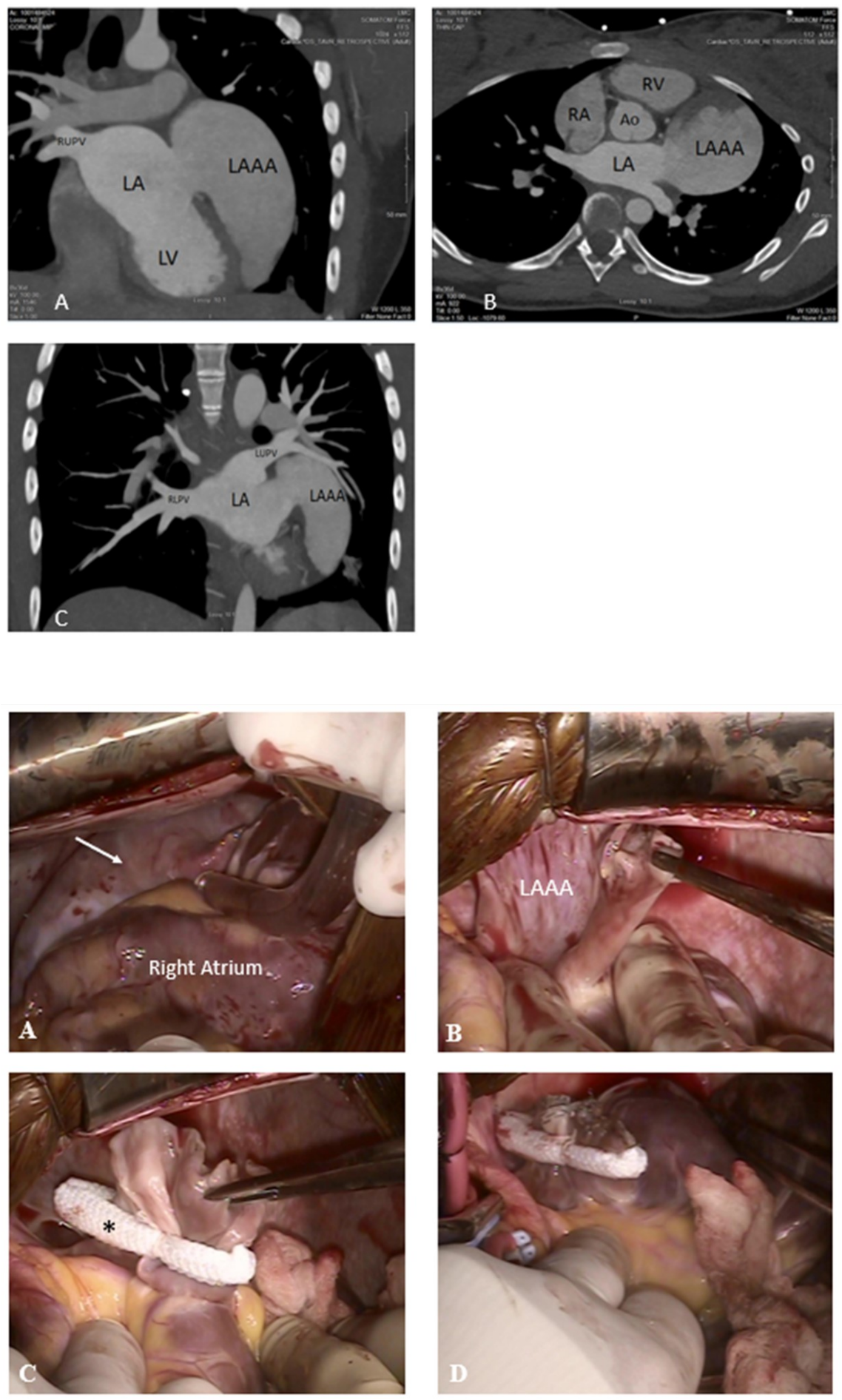